\title{
Prediction of malignancy in main-duct intraductal papillary mucinous neoplasm (MD-IPMN) based on radiologic features: How should we treat if main duct dilatation is under $10 \mathrm{~mm}$ ?
}

Hye-Sol JUNG, Youngmin HAN, Jae Seung KANG, Yoo Jin CHOI, Heeju SOHN, Jung Min LEE, Hongbeom KIM, Wooil KWON, Jin-Young JANG*

Department of Surgery and Cancer Research Institute, Seoul National University College of Medicine, Seoul, Korea

Introduction: Surgical indications of main-duct intraductal papillary mucinous neoplasm (MD-IPMN) except for main pancreatic duct (MPD) diameter $\geq 10 \mathrm{~mm}$ remain controversial. Diameter of MPD alone could result in overestimation of malignancy. We aimed to predict malignancy risk of MD-IPMN based on morphologic features presented in CT scan or MRI.

Methods: We retrospectively reviewed 258 patients with main-duct or mixed-type IPMN between 2000 and 2017 in our institute. MD-IPMN is defined as segmental or diffuse dilatation of MPD $>5 \mathrm{~mm}$ without other causes of obstruction. We divided MD-IPMN into focal ectatic and diffuse type by pattern of dilated MPD.

Results: Of 258 patients with main-duct involved IPMN, 47 (18.2\%) and 211 (81.8\%) had main-duct and mixed-type IPMN. Risk of malignant (high-grade dysplasia + invasive lesion) (66.0\% vs. $46.9 \% ; p=0.018)$ and invasive IPMN $(53.2 \%$ vs. $26.1 \% ; p<0.001)$ were higher in MD-IPMN than mixed-type. Patients with MPD $\geq 10 \mathrm{~mm}(\mathrm{n}=100)$ had more malignant $(64.0 \% \mathrm{vs.} 41.8 \% ; p<0.001)$ and invasive (43.0\% vs. $23.4 \%$; $p<0.001)$ lesion than those with MPD 5-9 mm ( $\mathrm{n}=158)$. Of 47 patients with MD-IPMN, focal ectatic and diffuse type were presented in 27 (57.4\%), and 20 (42.6\%). Forty three patients (91.5\%) showed MPD $\geq 10$ mm. Mean MPD diameter (maximal) was $17.4 \pm 12.2 \mathrm{~mm}$ in MD-IPMN group. Diffuse type had more invasive carcinoma compared with focal ectatic type (70\% vs. $40.7 \% ; p=0.047)$. Five-year survival was worse in diffuse type $(85.0 \%$ vs. $52.1 \% ; p=0.011)$. Focal ectatic MD-IPMN and mixedtype IPMN did not significantly differ in risk of malignant $(55.6 \%$ vs. $47.4 \%$; $p=0.425)$ lesion and in 5 -year survival $(85.0 \%$ vs. $77.6 \%$; $p=0.995)$. Symptoms $(p=0.011)$, CA19-9 $>37 \mathrm{IU} / \mathrm{mL}(p=0.014), \mathrm{MPD} \geq 10 \mathrm{~mm}(p=0.017)$, thickened cyst wall $(p=0.005)$, and distal atrophy $(p=0.015)$ were independent predictive factors for malignant IPMN.

Conclusions: Malignancy risk increased proportionally to the diameter of MPD in MD-IPMN. Those with above mentioned risk factors should be the candidate of surgery. 